\title{
RISCOS DA INTERAÇÃO DROGA-NUTRIENTE EM IDOSOS DE INSTITUIÇÃO DE LONGA PERMANÊNCIA
}

\author{
Jessica Sereno PEIXOTO ${ }^{a}$, Maria Aparecida SALCI ${ }^{\mathrm{b}}$, Cremilde Aparecida Trindade RADOVANOVIC ${ }^{\mathrm{c}}$, \\ Tania Pereira SALCI ${ }^{d}$, Maricy Morbin TORRES ${ }^{e}$, Lígia CARREIRA ${ }^{f}$
}

\section{RESUMO}

O objetivo do estudo foi verificar riscos da interação droga-nutriente nos idosos residentes em Instituição de Longa Permanência. Trata-se de estudo descritivo, de abordagem quantitativa, realizado em 73 idosos. A coleta dos dados ocorreu em 2008, através da análise dos prontuários, história dietética e avaliação do IMC. Os dados evidenciaram que os medicamentos mais utilizados foram do sistema nervoso e cardiovascular, totalizando $66 \%$ das prescrições. Entre 375 medicamentos prescritos, 166 fazem algum tipo de interação, 32,0\% diminuem o efeito de absorção do fármaco quando há utilização de cafeína e 14,3\% diminuem absorção de vitamina B12. A utilização de diversos medicamentos de uso contínuo pode acarretar prejuízo na absorção de nutrientes, aumentando o risco de desnutrição em idosos. Torna-se indispensável a ação da equipe de saúde, através da avaliação criteriosa dos medicamentos administrados, dietoterapia e a interação entre os mesmos, para beneficiar idosos com melhor aproveitamento da terapêutica e melhoria das condições nutricionais.

Descritores: Saúde do idoso. Interações alimento-droga. Instituição de longa permanência para idosos.

\section{RESUMEN}

El objetivo fue verificar el riesgo de interacciones fármaco-nutriente en los ancianos residentes en Institución de Larga Permanencia. Estudio descriptivo, cuantitativo, realizado en 73 ancianos. La recolección de datos se realizó en 2008 a través del análisis de los registros médicos, historia dietética y la evaluación del IMC. Los datos evidenciaron que los medicamentos más utilizados fueron del sistema nervioso y cardiovascular, totalizando el 66\% de las prescripciones; entre los 375 medicamentos prescriptos, 166 hacen algún tipo de interacción, el 32,0\% disminuye el efecto de absorción del fármaco cuando hay utilización de cafeina y el 14,3\% disminuye la absorción de vitamina B12. La utilización de diversos medicamentos de uso continuo puede acarrear perjuicio en la absorción de nutrientes. Vuelve indispensable la acción del equipo de salud a través de una cuidadosa evaluación de los medicamentos, la dieta y la interacción entre ellos, para beneficiar a los ancianos con mejor aprovechamiento de la terapéutica.

Descriptores: Salud del anciano. Interacciones alimento-droga. Hogares para ancianos.

Título: Riesgos de interacción droga-nutriente en ancianos de institución de larga permanencia.

\section{ABSTRACT}

This study was aimed at verifying the risks of drug-nutrient interactions in the elderly residents of a Long-Term Care Institution. Descriptive study of quantitative approach, performed in 73 elderly people. Data collection occurred in 2008 through analysis of medical records, diet history and evaluation of the BMI. Data evidenced that the drugs more frequently used were the ones for nervous and cardiovascular systems, totaling approximately 66\% of the prescriptions; among the 375 drugs prescribed, 166 make some type of interaction, 32.0\% reduce the effect of drug absorption when there is use with caffeine and $14.3 \%$ reduce the $B 12$ vitamin absorption. Taking several drugs of continuous use may cause damage to the absorption of nutrients. The action of the health team becomes vital, through careful evaluation of the administered drugs, diet and interaction between them, to benefit the elderly with a better use of the therapeutics and improvement of the nutritional conditions.

Descriptors: Elderly health. Food-drug interactions. Long-term care institutions for the elderly.

Title: Risks of drug-nutrient interaction for the elderly in long-term care institutions.

a Nutricionista, Mestre em Ciências da Saúde pelo Programa de Pós-Graduação em Ciências da Saúde da Universidade Estadual de Maringá (PCS - UEM), Maringá, Paraná, Brasil.

b Enfermeira, Doutorando em Enfermagem pelo Programa de Pós-Graduação em Enfermagem da Universidade Federal de Santa Catarina (UFSC), Professora Assistente do Departamento de Enfermagem da UEM, Maringá, Paraná, Brasil.

c Enfermeira, Doutorando em Ciências da Saúde pelo PCS da UEM, Professora Assistente do Departamento de Enfermagem da UEM, Maringá, Paraná, Brasil.

d Farmacêutica, Mestre pelo Programa de Pós-Graduação em Biociências Aplicadas à Farmácia da UEM, Maringá, Paraná, Brasil.

e Enfermeira, Mestre em Enfermagem, Professora Assistente do Departamento de Enfermagem da UEM, Maringá, Paraná, Brasil.

f Enfermeira, Doutora em Enfermagem, Professora Adjunto do Departamento de Enfermagem e do Programa de Pós-Graduação em Enfermagem da UEM, Maringá, Paraná, Brasil. 


\section{INTRODUÇÃO}

A crescente expectativa de vida e, consequentemente, o aumento do número de idosos, demonstram que a distribuição etária da população mundial tem apresentado visível alteração nas últimas décadas. O Brasil, assim como muitos países, está passando por um processo de envelhecimento rápido e intenso, deste modo, tal situação requer novos desafios no campo da pesquisa nutricional, pois, apesar deste ser um processo natural, o organismo é submetido a diversas alterações fisiopatológicas com repercussões na manutenção do estado nutricional, bem como na condição de saúde do idoso ${ }^{(1)}$.

Desta forma, a população idosa merece, cada vez mais, atenção dos órgãos públicos e da sociedade, enfatizando suas características sociais, econômicas e de saúde em cada região do território nacional. A prevalência de doenças crônicas aumentará, pois são frequentes neste grupo etário, o que demanda transferência de ênfase dos programas governamentais de saúde que visam à cura e sobrevivência, para melhora do estado funcional e do bem-estar do idoso ${ }^{(1)}$.

Sabe-se ainda que em função das morbidades crônicas presentes na população idosa e do consequente consumo elevado de medicamentos, esta constitui grupo de risco para a ocorrência de reações adversas aos medicamentos, interações medicamentosas e outros efeitos em decorrência, principalmente, da utilização de polifarmácia ${ }^{(1)}$.

Possivelmente, indivíduos com múltiplas doenças, como disfunção renal ou hepática, bem como aqueles que fazem uso de muitos medicamentos são os mais suscetíveis a experimentar reações de interações medicamentosas adversas, situações estas nas quais o idoso, geralmente, se encaixa. A interação medicamentosa é um evento clínico em que os efeitos de um fármaco ficam alterados pela presença de outro fármaco, fitoterápico, alimento, bebida alcoólica ou agente químico ambiental ${ }^{(1)}$.

Além disso, o processo de envelhecimento leva a um progressivo déficit da reserva funcional de múltiplos órgãos e sistemas, influenciando a farmacocinética e farmacodinâmica dos medicamentos, principalmente na biotransformação hepática e excreção renal, podendo aumentar a predisposição à toxicidade relacionada ao uso de fármacos e a ocorrência de interações medicamentosas ${ }^{(1,2)}$.

Neste contexto, o estado nutricional também tem importantes implicações, visto que o controle de grande parte das doenças crônicas ou infecciosas, além da prevenção de complicações decorrentes das mesmas, dependem do bom estado nutricional do idoso ${ }^{(1)}$. Para além destes aspectos, podemos destacar que as interações entre nutrientes e o uso de medicamentos em idosos, provocam grande impacto farmacológico devido às alterações fisiológicas e efeitos deletérios de drogas no estado nutricional ${ }^{(1)}$.

Apesar de compreendermos a importância desse conhecimento na condução da terapêutica do idoso, no Brasil parece haver uma lacuna na literatura sobre pesquisas com essa população asilada e a relação do uso de medicamentos e a alimentação, analisando os riscos da interação entre drogas e nutrientes para a saúde do idoso. Evidencia-se, desse modo, a necessidade de estudos nessa área, que investiguem esta temática com intuito de ampliar informações na geriatria brasileira. Diante do exposto, o estudo teve como objetivo verificar riscos da interação droga-nutriente nos idosos residentes em uma Instituição de Longa Permanência (ILP) da região noroeste do Paraná.

\section{MATERIAIS E MÉTODOS}

Trata-se de estudo descritivo, de abordagem quantitativa, realizado com todos os idosos de uma ILP localizada no noroeste do Estado do Paraná, totalizando 73 residentes. Esta instituição asilar tem caráter filantrópico e existe desde 1984, a qual é mantida com parte dos benefícios monetários dos idosos moradores, doações da comunidade e também conta com auxílio da prefeitura municipal através de contratação de profissionais que realizam os cuidados nos internos.

A pesquisa foi realizada no período de março a julho de 2008. Foi critério de inclusão residir na ILP e concordar com a participação no estudo. Para coleta de dados buscou-se informações referentes aos medicamentos utilizados pelos idosos e a alimentação oferecida para os mesmos, ocorrendo nesta etapa a participação de todos os idosos. Os dados relativos aos medicamentos foram consultados nos 73 prontuários dos idosos, a fim de conhecer suas prescrições médicas, investigou-se: nome dos fármacos, posologia (dose e frequência) e modo de utilização (horários e uso com alimentação). Informações complementares sobre as prescrições foram obtidas com a equipe de enfermagem.

Quanto às informações referentes à alimentação fornecida aos idosos, foi aplicado um questionário ao responsável pela instituição e também aos manipula- 
dores de alimentos do refeitório, contendo perguntas referentes ao cardápio oferecido, frequência alimentar, bem como os horários das refeições servidas.

Realizou-se ainda a caracterização do perfil dessa população através da identificação do sexo, idade, peso, altura, tempo de permanência na instituição e índice de massa corporal (IMC), que tem por finalidade avaliar a relação altura e peso do indivíduo, o mesmo foi calculado a partir do peso (em quilograma) dividido pelo quadrado da altura (em metros). A identificação do IMC foi realizada somente em 60 idosos, pois houve recusa de nove participantes nesta avaliação e quatro idosos encontravam-se acamados, o que impossibilitou a manipulação necessária para esta análise.

$\mathrm{Na}$ análise dos dados utilizou-se a classificação dos fármacos em subgrupo terapêutico, de acordo com a classificação Anatômica-Terapêutica-Clínica (ATC), proposta pela Organização Mundial da Saúde $(\mathrm{OMS})^{(1)}$. A partir da classificação dos fármacos e da análise de suas possíveis interações com os nutrientes, utilizou-se a estatística descritiva.

Em relação aos aspectos éticos, foram respeitados os preceitos contidos na Resolução n. 196/96 do Conselho Nacional de Saúde do Ministério da Saúde. Todos entrevistados foram orientados sobre a pesquisa e foi solicitada assinatura do Termo de Consentimento Livre e Esclarecido. O projeto foi avaliado e recebeu parecer favorável do Comitê de Ética (COPEP) da Universidade Estadual de Maringá (parecer n.131/2008).

\section{RESULTADOS}

\section{Caracterização dos idosos e uso de medicamentos}

O tempo médio de permanência dos idosos na ILP foi de 5 anos, com variação mínima entre um mês e máxima de 24 anos. Os idosos possuíam média de idade de 74,8 anos, variando com idade mínima de 60 e máxima de 101 anos, dos quais 57,5\% eram do sexo masculino e $42,4 \%$ do sexo feminino.

$\mathrm{O}$ número de medicamentos consumidos por idoso variou entre 1 a 13 tipos, num total de 345 medicamentos prescritos. A média de medicamentos utilizados por pessoa ao dia foi de 4,7. Observou-se que $26 \%$ dos idosos utilizavam de 1 a 3 medicamentos e $74 \%$ usavam 4 ou mais medicamentos. Todos os medicamentos foram prescritos pelo médico da instituição e administrados pela equipe de enfermagem.

Os medicamentos mais utilizados, reunidos por grupamento anatômico segundo classificação $\mathrm{ATC}^{(9)}$, foram do sistema nervoso $(37,8 \%)$ e do aparelho cardiovascular (28,0\%), totalizando $65,8 \%$ das prescrições. Os medicamentos do aparelho

\begin{tabular}{|c|l|c|}
\hline & \multicolumn{1}{|c|}{ Categoria* } & \% (n) \\
\hline A & Aparelho disgestório e metabólico & $18,5(64)$ \\
B & Sangue e órgão hematopoiéticos & $11,0(38)$ \\
C & Aparelho cardiovascular & $\mathbf{2 8 , 0 ( 9 6 )}$ \\
D & Terapia dermatológica & $1,2(4)$ \\
G & Terapia geniturinária (incluindo hormônios sexuais) & $0,0(0)$ \\
H & Terapia hormonal & $0,3(1)$ \\
J & Terapia antiinfecciosa (uso sistêmico) & $1,2(4)$ \\
L & Terapia antineoplásica e agentes imunomoduladores & $0,0(0)$ \\
M & Sistema musculoesquelético & $1,4(5)$ \\
N & Sistema nervoso & $\mathbf{3 7 , 6}(\mathbf{1 3 0})$ \\
P & Antiparasitários, inseticidas e repelentes & $0,0(0)$ \\
R & Aparelho respiratório & $0,8(3)$ \\
S & Órgãos dos sentidos & $0,0(0)$ \\
V & Vários & $0,0(0)$ \\
\hline & Total & $\mathbf{1 0 0 , 0}(\mathbf{3 4 5})$ \\
\hline
\end{tabular}

Quadro 1-Categorias de medicamentos prescritos por grupamento anatômico (n=345). Maringá, PR, 2008.

Fonte: Prontuários dos idosos da ILP.

* Grupamentos segundo classificação ATC (Anatômica-Terapêutica-Química)(9). 
digestório e metabólico representaram 18,6\% das prescrições. O quadro 1 mostra detalhadamente a distribuição dos medicamentos reunidos segundo grupamento anatômico.

No grupamento referente ao sistema nervoso, os fármacos mais utilizados corresponderam ao subgrupo dos antipsicóticos (28,4\%), antiepilépticos/ anticonvulsivantes (18,5\%), antidepressivos (15,3\%) e calmantes (7,0\%). No grupamento equivalente ao aparelho cardiovascular, os subgrupos terapêuticos mais frequentes foram os anti-hipertensivos (69,8\%).

De um modo geral, entre os medicamentos utilizados pelos idosos, destacou-se o ácido acetilsalicílico com maior número de prescrições (8,12\%), seguido da hidroclorotiazida (5,80\%), haloperidol $(5,51 \%)$ e captopril $(4,93 \%)$.

\section{Índice de Massa Corporal dos idosos institucionalizados}

A média em relação à estatura nos anciãos da unidade asilar analisada foi de 1,54 m. No que se refere ao peso, a média encontrada foi de $56,0 \mathrm{~kg}$. Os homens obtiveram média de peso maior, 58,8 $\mathrm{kg}$, em relação às mulheres que apresentaram mé- dia de peso de 54,0 kg. Idosos do sexo masculino obtiveram 63,3\% de peso adequado, valor este maior quando comparado ao das mulheres que obtiveram 40,0\% apenas para classificação de peso normal.

O valor médio do IMC observado nesta pesquisa foi de $23,5 \mathrm{~kg} / \mathrm{m}^{2}$, considerado dentro da normalidade para essa população. Entretanto, 16,6\% dos idosos do sexo masculino encontravam-se abaixo do peso e $10 \%$ estavam em obesidade. Em comparação, os valores do IMC encontrados entre as mulheres mostraram índice mais elevado tanto para classificação de baixo peso quanto a obesidade (26,6\%). A tabela 1 demonstra detalhadamente a classificação das categorias do IMC dos idosos segundo sexo e faixa etária.

Ao analisar as mulheres individualmente, observa-se que a maioria apresentou alteração do IMC, sendo $26,6 \%$ com baixo peso, $6,6 \%$ sobrepeso e $26,6 \%$ obesas.

\section{Interação droga-nutriente em idosos institucionalizados}

Os 345 medicamentos prescritos foram compostos por 87 diferentes fármacos, destes 37 (42,5\%) não possuem interação com nutrientes, 22 (25,3\%)

Tabela 1 - Distribuição das categorias do IMC dos idosos institucionalizados, segundo faixa etária e sexo (n=60). Maringá, PR, 2008.

\section{IMC}

\begin{tabular}{|c|c|c|c|c|}
\hline $\begin{array}{c}\text { Faixa Etária } \\
\text { (anos) }\end{array}$ & $\begin{array}{c}\text { Baixo peso } \\
\text { n }(\%)\end{array}$ & $\begin{array}{c}\text { Adequado } \\
\text { n (\%) }\end{array}$ & $\begin{array}{c}\text { Sobrepeso } \\
\text { n (\%) }\end{array}$ & $\begin{array}{c}\text { Obeso } \\
\text { n }(\%) \\
\end{array}$ \\
\hline \multicolumn{5}{|l|}{ Masculino } \\
\hline $60-69,9$ & $1(0,3 \%)$ & $7(23,3 \%)$ & $1(0,3 \%)$ & $2(6,6 \%)$ \\
\hline $70-79,9$ & --- & $9(30 \%)$ & $2(6,6 \%)$ & ---- \\
\hline $80-89,9$ & $3(10 \%)$ & $2(6,6 \%)$ & --- & $1(0,3 \%)$ \\
\hline 90 e mais & $1(0,3 \%)$ & $1(0,3 \%)$ & ---- & ---- \\
\hline Total & $5(16,6 \%)$ & $19(63,3 \%)$ & $3(10 \%)$ & $3(10 \%)$ \\
\hline \multicolumn{5}{|l|}{ Feminino } \\
\hline $60-69,9$ & $3(10 \%)$ & $2(6,6 \%)$ & $1(0,3 \%)$ & $4(13,3 \%)$ \\
\hline $70-79,9$ & $4(13,3 \%)$ & $2(6,6 \%)$ & $1(0,3 \%)$ & $2(6,6 \%)$ \\
\hline $80-89,9$ & $1(0,3 \%)$ & $7(23,3 \%)$ & --- & $1(0,3 \%)$ \\
\hline 90 e mais & ---- & $1(0,3 \%)$ & ---- & $1(0,3 \%)$ \\
\hline Total & $8(26,6 \%)$ & $12(40 \%)$ & $2(6,6 \%)$ & $8(26,6 \%)$ \\
\hline
\end{tabular}


medicamentos não foram encontrados informações relacionando interações dos mesmos com os alimentos e 28 (32,0\%) fazem algum tipo de interação droga-nutriente, sendo identificados em 166 prescrições.

Entre os fármacos que possuem interação droga-nutriente, nove (32,0\%) diminui o efeito de absorção do fármaco quando há consumo de cafeína (xantina); quatro $(14,3 \%)$ diminui a absorção de vitamina B12 e dois $(7,1 \%)$ diminui a absorção do fármaco quando utilizado suplemento com cálcio. A relação completa da interação dos medicamentos com os nutrientes está evidenciada na Tabela 2.

Tabela 2 - Relação de medicamentos que interagem com nutrientes (n=28). Maringá, PR, 2008.

\begin{tabular}{|c|c|c|}
\hline Medicamentos & Interações droga-nutrientes* & $\%(n)$ \\
\hline $\begin{array}{l}\text { Diazepam } \\
\text { Nortriptilina } \\
\text { Imipramida } \\
\text { Ormigrein } \AA \\
\text { Pentoxifilina } \\
\text { Lorazepam } \\
\text { Haloperidol } \\
\text { Ranitidina } \\
\text { Clomipramida }\end{array}$ & Limitar cafeína (xantina) & $32,0(9)$ \\
\hline $\begin{array}{l}\text { Clorpromazina } \\
\text { Metildopa } \\
\text { Metformina } \\
\text { Omeprazol }\end{array}$ & Diminui a absorção de vitamina B12 & $14,3(4)$ \\
\hline $\begin{array}{l}\text { Captopril } \\
\text { Sulfato ferroso }\end{array}$ & $\begin{array}{l}\text { Tomados com alimentos reduz a absorção do fármaco de } 30 \\
\text { a } 50 \%\end{array}$ & $7,1(2)$ \\
\hline 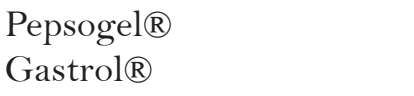 & Terapia quelante de fosfato & $7,1(2)$ \\
\hline Sulfametaxazol & Interfere no metabolismo do folato (ácido fólico) & $3,6(1)$ \\
\hline Fenobarbital & $\begin{array}{l}\text { Aumenta a necessidade de vitamina } \mathrm{C} \text { e aumenta a taxa de } \\
\text { metabolismo da vitamina } \mathrm{K} \text { e } \mathrm{D}\end{array}$ & $3,6(1)$ \\
\hline $\begin{array}{l}\text { Fenitoína } \\
\text { Propranolol }\end{array}$ & Suplementos com cálcio diminuem a absorção do fármaco & $7,1(2)$ \\
\hline Digoxina & Uso de muitas fibras diminui a absorção do fármaco em $25 \%$ & $3,6(1)$ \\
\hline AAS(ácido acetilsalicílico) & Diminui a absorção de alimentos & $3,6(1)$ \\
\hline $\begin{array}{l}\text { Hidroclorotiazida } \\
\text { Diupress }{ }^{\circledR}\end{array}$ & Suplementos de cálcio e ou vitamina D: risco de hipercalcemia & $7,1(2)$ \\
\hline Levotiroxina sódica & $\begin{array}{l}\text { Tomar suplementação de Fe separadamente em } 4 \text { h pode di- } \\
\text { minuir a absorção }\end{array}$ & $3,6(1)$ \\
\hline Bisacodil & Diminui a absorção de aminoácidos e glicose & $3,6(1)$ \\
\hline Alopurinol & $\begin{array}{l}\text { Doses altas de vitamina } \mathrm{C} \text { aumentam a potencialidade de } \\
\text { cálculos renais }\end{array}$ & $3,6(1)$ \\
\hline Total & & $100,0(28)$ \\
\hline
\end{tabular}


Os medicamentos eram administrados em horários padronizados pela instituição, os quais estavam estabelecidos para as sete horas e dez horas da manhã, as duas e quatro horas da tarde e dez horas da noite. Vale ressaltar que esses horários não coincidiam com o momento de servir as refeições.

As refeições realizadas pelos idosos eram fracionadas em seis vezes ao dia (café da manhã, lanche da manhã, almoço, lanche da tarde, jantar e ceia) com intervalos de aproximadamente três horas.

O cardápio semanal abrangia frutas, café, chá e pão com margarina para os lanches da manhã e tarde, arroz, feijão, carne e salada no almoço e sopas no jantar; chá e café eram oferecidos três vezes ao dia e frutas em duas refeições. Nem todos os idosos realizavam as seis refeições oferecidas.

\section{DISCUSSÃO}

Neste estudo, observamos um longo tempo de permanência de alguns idosos na instituição asilar, revelando a precocidade dos internamentos. Esse fato pode ser atribuído à insuficiente integração de pessoas senis à sociedade e a falta de estrutura familiar, na qual optam pelo internamento como um recurso para atender as necessidades de cuidado aos idosos ${ }^{(2)}$. Dessa forma, vê-se cada vez mais a necessidade de melhorias nesses lares para que possam ser fontes promotoras de uma forma de vida saudável a essas pessoas.

Nos idosos as condições nutricionais são associadas a diversas mudanças fisiológicas próprias da senescência, tais como perdas sensoriais, alterações da boca (higiene e perda de dentes), variações na função gastrintestinal, mudanças renais e diminuição da imunidade natural. Esses fatores aliados às condições de saúde física e mental, classe social e relações psicoafetivas podem levar a alterações nutricionais, que compreende tanto a desnutrição como a obesidade, sendo que a primeira é mais frequentemente observada em indivíduos institucionalizados ${ }^{(2,1)}$.

O IMC é utilizado como critério para avaliar o estado nutricional de indivíduos ${ }^{(2)}$, o valor considerado ideal para idosos compreende 18,50 a $24,99 \mathrm{Kg} / \mathrm{m}^{2}$. Entre os idosos avaliados, a maioria apresentou índices condizentes com os valores considerados ideais, no entanto na população feminina as variações para sobrepeso e obesidades foram mais significativas que entre os homens.
Para amenizar problemas referentes à má nutrição, estudos nutricionais com idosos sugerem que as refeições sejam oferecidas de modo atrativo, tomando-se atitudes simples como servir a alimentação em locais agradáveis e na presença de boas companhias $^{(1)}$. Outra medida que também pode ser eficaz é a implantação de programa de orientação de atividade física e nutricional, que engloba não somente indicações sobre alimentação saudável, mas também aconselhamentos sobre atividade física, com intuito de contribuir para melhoria da qualidade de $\operatorname{vida}^{(2)}$.

Sabe-se que o uso prolongado de medicamentos pode ser um dos fatores que favorecem a perda de nutrientes, como é o caso dos tratamentos de doenças crônicas, usualmente existentes na população idosa. O número de medicamentos prescritos na instituição asilar foi significativo, com uma média de 4,7 medicamentos por pessoa. A prescrição medicamentosa para o idoso é maior quando comparada com outras faixas etárias, em virtude da multimorbidade, o que eleva o risco de indução da deficiência nutricional quando a prescrição ultrapassa três medicamentos, sendo necessário, nestes casos, a suplementação dietética para restabelecer as condições nutricionais normais da pessoa ${ }^{(2)}$.

Tomando-se consciência das necessidades desses indivíduos, destacamos o cuidado básico com alimentação e terapias medicamentosas utilizadas. $\mathrm{Na}$ população em estudo ficou evidente a constituição de polifarmácia, sendo que dentre o número de fármacos prescritos, aproximadamente metade $(48,1 \%)$ tem risco de fazer algum tipo de interação droga-nutriente.

A farmacoterapia é comum em idosos e o conhecimento do potencial das interações entre drogas e nutrientes pode permitir intervenções que previnam efeitos colaterais indesejáveis, limitando a terapia medicamentosa indicada, ou elaborando estratégias para melhoria da escolha dos nutrientes, desse modo pode-se evitar os efeitos adversos que contribuem para a perda de peso e consequente risco de desnutrição( ${ }^{(3)}$.

Os medicamentos utilizados pelos idosos podem ser mais ou menos absorvidos se associados ou não às refeições, o que depende também da condição nutricional do mesmo; isso porque com o envelhecimento ocorre mudanças nos processos farmacocinéticos do indivíduo ${ }^{(6)}$. Por outro lado, as deficiências nutricionais podem ocorrer por indução medicamentosa, sendo mais frequentes as depleções de vitaminas e minerais ${ }^{(16)}$. 
Ressalta-se que a deficiência nutricional é um problema relevante nessa população, devido alterações fisiológicas, medicamentosas e dietéticas que influenciam nesse aspecto, podendo aumentar o risco de desnutrição que pode ser devida a alterações características do processo de envelhecimento, do efeito deletério das drogas no estado nutricional e da interação entre nutrientes e medicamentos ${ }^{(8)}$.

A população em questão encontra-se sob cuidados profissionais constantes, o que facilita o controle do uso de medicamentos e da nutrição por pessoas capacitadas que devem analisar o conteúdo alimentar bem como os horários da tomada de medicamentos, de forma a corrigir os erros significativos e aumentar a possibilidade de bons resultados terapêuticos.

Algumas interações medicamentosas com nutrientes são descritas na literatura científica como, por exemplo, os componentes minerais que podem competir entre si, durante o processo absortivo, como por exemplo, zinco, ferro, anticoagulante e antibiótico pode induzir a deficiência de vitamina K; antiácidos podem alterar a absorção de ferro, cálcio e vitamina B 12; neomicina, colchicina, colestiramina e clindamicina, dietas hiperprotéicas diminuem a atividade de agentes dopaminérgicos utilizados no tratamento da doença de Parkinson(2).

No presente estudo, evidenciou-se que a maioria dos fármacos presentes nas prescrições (Diazepam, Nortriptilina, Imipramida, Ormigrein, Pentoxifilina, Lorazepam, Haloperidol, Ranitidina e Clomipramida) possui interação droga-nutriente relacionada com a limitação quanto ao uso de cafeína devido à diminuição da ação dos mesmos. A ingestão de 100-200mg de cafeína é suficiente para causar interações significativas ${ }^{(11)}$. Chá, café, chocolate e alguns tipos de refrigerantes são exemplos de fontes alimentares ricas em cafeína, sendo que o café-grão torrado (xícara com $150 \mathrm{ml}$ ) possui 103mg de cafeína ${ }^{(2)}$, a qual é uma das bebidas mais consumidas entre os idosos. Além de que, a cafeína pertence à família dos compostos químicos chamados xantinas, essa substância estimula o sistema nervoso central e músculo cardíaco, como diurético e relaxante muscular.

O grupamento do sistema nervoso foi evidenciado quanto à grande utilização de fármacos antipsicóticos, seguido de antidepressivos e calmantes. Sabe-se que o sistema nervoso é o sistema biológico mais comprometido com o processo do envelhecimento, por ser responsável pelas relações durante a vida, tais como sensações, movimentos, funções psíquicas, e ainda responde pela vida vegetativa, a qual envolve as funções biológicas internas do organismo ${ }^{(2)}$.

Além disso, os idosos podem sofrer efeitos adversos do uso desses medicamentos, sendo mais frequentes os tranquilizantes e psicofármacos que favorecem o relaxamento e diminuem a absorção intestinal ${ }^{(10)}$. A utilização de fenobarbital que tem como principal indicação o tratamento da epilepsia, em longo prazo pode levar a uma necessidade de suplementação com vitamina $\mathrm{D}, \mathrm{B} 12$, folato e vitamina $\mathrm{C}$, além de elevar a taxa de metabolismo da vitamina $\mathrm{D}$ e $\mathrm{K}^{(11)}$.

Estudos realizados no Brasil ${ }^{(2,3)}$, investigando o uso de medicamentos em idosos que residem em domicílios, identificaram como prevalente o consumo de drogas da categoria C (aparelho cardiovascular). Ao contrário, no presente estudo houve predominância de utilização dos medicamentos da categoria N (sistema nervoso), no entanto devemos levar em consideração que se trata de idosos institucionalizados.

Dentre os medicamentos da categoria C, o ácido acetilsalicílico foi o medicamento mais prescrito para os idosos, o qual é administrado, principalmente, com a finalidade antitrombótica, sendo frequentemente administrados com alimentos, com o objetivo de diminuir as irritações da mucosa gástrica, provocadas pelo uso prolongado ${ }^{(16)}$. Porém, este medicamento diminui a absorção de alimentos e a sua utilização a longo prazo requer um aumento de alimentos ricos em vitamina $\mathrm{C}$ e ácido fólico ${ }^{(11)}$.

A hidroclorotiazida também apresentou número significativo de prescrições. Esse medicamento é utilizado pela ação farmacológica diurética e anti-hipertensiva. Os diuréticos e laxantes ocasionam desidratação e depleção de eletrólitos como magnésio, potássio e zinco ${ }^{(10)}$.

Outro fármaco bastante administrado para os idosos foi o captopril, pertencente à categoria do aparelho cardiovascular. Recomenda-se que este seja ingerido com estômago vazio (uma hora antes das refeições), pois o alimento diminui sua absorção em $30-50 \%$. Estudo que avaliou riscos de problemas relacionados com medicamentos em pacientes de uma instituição geriátrica destacou o uso desse medicamento com prevalência de $22,8 \%$ durante as refeições, prejudicando assim sua biodisponibilida$\mathrm{de}^{(12)}$. No entanto, no presente estudo o captopril, bem como os outros fármacos, foi administrado 
corretamente aos idosos, ou seja, longe do horário das refeições evitando perda de absorção do mesmo.

$\mathrm{O}$ sulfato ferroso pode ser ingerido com alimentos, porém, neste caso, há uma redução de 50\% em sua absorção, indicando-se a administração uma hora antes ou duas horas após alimentos ricos em fibras e ou fitatos, chá ou café. Na alimentação é importante utilizar $200 \mathrm{mg}$ de vitamina C e $30 \mathrm{mg}$ de ferro para aumentar sua absorção ${ }^{(11)}$.

A digoxina, utilizada no tratamento da insuficiência cardíaca congestiva, possui propriedade anorexígena, além de causar náuseas e vômitos. O uso concomitante com diurético facilita a perda não somente de sódio, mas também de potássio, magnésio e cálcio ${ }^{(16)}$. A digoxina foi administrada associada ao diurético em cinco pacientes idosos, isso requer mais atenção com as perdas de micronutrientes como cálcio, mineral de extrema importância a população idosa, essencial para manutenção óssea e na prevenção da osteoporose.

Antiácidos devem ser ingeridos de uma a três horas após as refeições, pois se trata de uma terapia quelante de fosfato. A ingestão de sucos e frutas cítricas deve ser feita separadamente em intervalos de três horas após sua administração, pois esses aumentam a absorção de alumínio e diminui absorção de ácido fólico, fósforo e ferro ${ }^{(11)}$.

Embora os antibióticos não sejam de uso contínuo, são utilizados pela população idosa com certa frequência, em virtude das condições de susceptibilidade a infecções impostas pelo processo de envelhecer. Quando consumidos, esse grupo de fármacos altera absorção intestinal por destruição da flora natural, provocando má absorção de carboidratos, vitamina $\mathrm{B}_{12}$, cálcio, ferro, magnésio e cobre, inibindo a síntese proteica; já os glicocorticóides predispõem à gastrite, osteoporose e hiperglicemia, e os analgésicos favorecem gastrite e úlceras ${ }^{(10)}$.

Em alguns casos, os medicamentos devem ser administrados longe das refeições, isso porque têm a capacidade de diminuir a velocidade de absorção dos fármacos por retardarem o esvaziamento gástrico ou provocar interações que induzam outros danos ao organismo. Porém, ressalta-se que alguns medicamentos devem ser ingeridos próximos às refeições, para que não agridam a mucosa gastrointestinal, possibilite aumento de sua absorção ou também seja usado como auxiliar no cumprimento da terapia ${ }^{(16)}$.

Cuidados com o intervalo de tempo entre a ingestão de fármacos e alimentos são determinantes na terapêutica, pois podem afetar principalmente a absorção dos mesmos, influenciando a velocidade e magnitude da biodisponibilidade das drogas ${ }^{(15,17)}$.

\section{CONSIDERAÇÕES FINAIS}

Tendo em vista a longa permanência do idoso nas instituições asilares, estas devem ter ciência das necessidades de seus moradores no que tange a boa qualidade de vida, o que inclui além dos cuidados básicos diários oferecidos por uma equipe de enfermagem qualificada, boas condições nutricionais e assistência farmacêutica.

A necessidade nutricional do indivíduo idoso pode ser comprometida quando associada às alterações próprias do processo de senescência. Além disso, com o processo de adoecer característico dessa fase do ciclo vital, é comum o consumo de diversos medicamentos de uso contínuo, o que pode acarretar prejuízo na digestão, absorção, excreção e biodisponibilidade de muitos nutrientes, aumentando o risco de interação droga-nutriente em idosos.

Assim, a alimentação e as condições que estas são oferecidas aos idosos nas ILPs são fatores essenciais na manutenção do estado de saúde dessa população. As instituições precisam atentar-se para esses aspectos, desenvolvendo estratégias que estimulem a boa alimentação e o prazer pelo consumo do alimento saudável, suprimindo as situações que geram efeito deletério através do uso de medicações.

Portanto, torna-se indispensável à ação da equipe de saúde multidisciplinar, devendo ser feita avaliação criteriosa não somente acerca dos múltiplos medicamentos administrados, mas também em relação à prescrição dietoterápica através dos dados da avaliação nutricional e da interação com as drogas utilizadas, atentando para os horários dos medicamentos e das refeições ofertadas. Dessa forma, poderemos beneficiá-los com melhor aproveitamento da terapêutica, além da melhoria das condições nutricionais, o que levará a consequente preservação do bom estado de saúde.

Por fim, apesar dos resultados encontrados neste estudo evidenciar a existência de riscos de interações entre droga-nutriente em uma proporção significativa de medicamentos consumidos pelos idosos da ILP e, ao mesmo tempo, apontar que cuidados tem sido tomados para evitar ou reduzir esses problemas, faz-se necessário ampliar este objeto de investigação em outros grupos de idosos 
fragilizados, para que seja possível identificar situações nocivas à saúde e indicar intervenções cabíveis para melhorar a vida dessa população.

\section{REFERÊNCIAS}

1 Garcia ANM, Romani SAM, Lira PIC. Indicadores antropométricos na avaliação nutricional de idosos: um estudo comparativo. Rev Nutr. 2007;20:371-8.

2 Jesus IS, Sena ELS, Meira EC, Gonçalves LHT, Alvarez AM. Cuidado sistematizado a idosos com afecções demencial residentes em instituição de longa permanência. Rev Gaúcha Enferm 2010;31(2):285-92.

3 Costa SC, Pedroso ERP. A prescrição de medicamentos para idosos internados em serviço de clínica médica: atualização Rev Med Minas Gerais. 2011;21(2):201-214.

4 Jacomini LCL; Silva NA. Interações medicamentosas: uma contribuição para o uso racional de imunossupressores sintéticos e biológicos. Rev Bras Reumatol. 2011;51(2):161-174.

5 Shi S, Morike K, Klotz U. The clinical implications of ageing for rational drug therapy. Eur J Clin Pharmacol. 2008;64:183-99.

6 Turnheim K. Drug therapy in the elderly. Exp Gerontol. 2004;39(11-12):1731-38.

7 Nascimento CM, Ribeiro AQ, Sant'Ana LFR, Oliveira RMS, Franceschini SCC, Priore SE. Estado nutricional e condições de saúde da população idosa brasileira: revisão da literatura. Rev Med Minas Gerais. 2011;21(2):174-180.

8 Pickering G. Frail elderly, nutricional status and drugs. Arch Gerontol Geriatr. 2004;38(2):174-80.

9 World health organization. Guidelines for ATC classification and DDD assignment. Oslo: WHO; Center for Drug Statistics Methodology; 2008. p. 269.

10 Cardoso SP, Martins C. Interações droga-nutriente. Curitiba: Nutroclínica; 1998.

\section{Endereço da autora / Dirección del autor / Author's address}

Lígia Carreira

Rua Pioneiro Cosme Gonçalves de Meireles, 190, Jardim Tóquio 87025-460, Maringá, PR

E-mail: ligiacarreira@hotmail.com
11 Correr CJ, Pontarolo R, Ferreira LC, Baptistão SAM. Riscos de problemas relacionados com medicamentos em pacientes de uma instituição geriátrica. Revista Brasileira de Ciências Farmacêuticas. Brazilian Journal of Pharmaceutical Sciences. 2007;43(1):55-62.

12 Castro MAC, Resrepo PAH, Ospina AMJ, Carmona SMR, Rueda LMS, Ruiz PAG, Yepes LMR, González JAR. Factores nutricionales, sociales, psicoafetivos y de salud de los adultos mayores. Perspect Nutr Hum. 2006; 15:67-82.

13 Pan American Health Organization, Central American Diabetes Initiative (CAMDI). Survey of diabetes, hypertension, and chronic disease risk factors, Belize City, Belize. Washington: PAHO/WHO; 2007.

14 Ferreira M, Matsudo S, Matsudo V, Braggion G. Efeitos de um programa de orientação de atividade física e nutricional sobre o nível de atividade física de mulheres fisicamente ativas de 50 a 72 anos de idade. Rev Bras Med Esporte. 2005;11(3):172-76.

15 Moura MRL, Reys FG. Interação fármaco-nutriente: uma revisão. Rev Nutr. 2002;15(2):223-238.

16 Marchini JS, Ferriolli E, Moriguti JC. Suporte nutricional no paciente idoso: definição, diagnóstico, avaliação e intervenção. Med. 1998;31:54-61.

17 Linder, M. Nutricional Biochemistry and metabolism witch clinical applications. $2^{\text {a }}$ ed. Nova York: Elsevier; 1991.

18 Cançado FAX, Horta ML. Envelhecimento cerebral. In: Freitas EV, Py L, Cançado FAX, Doll J, Gorzoni ML. Tratado de geriatria e gerontologia. Rio de Janeiro: Guanabara-Koogan; 2006. p.194-211.

19 Flores LM, Mengue SS. Uso de medicamentos por idosos em região sul do Brasil. Rev Saúde Públ. 2005;39(6):924-29.

20 Oliveira CAP, Marin MJS, Marchioli M, Pizoletto BHM, Santos RV. Caracterização dos medicamentos prescritos aos idosos na Estratégia Saúde da Família. Cad Saúde Públ. 2009;25(5):1007-16

Recebido em: 25.10.2011

Aprovado em: 12.07.2012 